\title{
ViMeNO: A Virtual Wireless Mesh Network Architecture for Operators
}

\author{
Katarzyna Kosek-Szott, Janusz Gozdecki, Krzysztof Loziak, Marek Natkaniec, \\ Szymon Szott and Michal Wagrowski \\ AGH University of Science and Technology, Faculty of Computer Science, Electronics and Telecommunications, \\ al. Mickiewicza 30, 30-059 Krakow, Poland \\ \{kosek, gozdecki, loziak, natkaniec, szott,wagrowski\}@kt.agh.edu.pl
}

\begin{abstract}
Keywords: Wireless Mesh Networks, Virtualization, Virtual Network Operators, Resource Separation.
Abstract: Network virtualization is one of the key concepts of the Future Internet. However, the use of virtualization techniques in wireless mesh networks has thus far not been adequately studied. In this paper we describe ViMeNO: a Virtual wireless Mesh Network architecture for Operators, i.e., the first virtualization-enabled wireless mesh network architecture designed for operators. In the proposed architecture, virtualization is used to divide the mesh resources governed by a single mesh network operator among multiple virtual network operators. The advantage of this outsourcing approach is that each virtual network operator can focus on providing a service to its end-users, leaving the network administration to the mesh network operator. This leads to the lowering of the operating expenditure of virtual network operators and allows tailoring of each virtual network to individual operator and service requirements.
\end{abstract}

\section{INTRODUCTION}

Network virtualization is one of the key concepts of the Future Internet. It enables decoupling the provided services from the underlying infrastructure (Chowdhury and Boutaba, 2010). There have been multiple research efforts devoted to this topic, mostly with respect to fixed core networks (4WARD, http://www.4ward-project.eu/; AKARI, http://akari-project.nict.go.jp/; ANA, http://www.anaproject.org/; GENI, http://www.geni.net/). In the domain of wireless local area networks, virtualization has been implemented at the interface level, allowing one WiFi access point (AP) to control multiple networks (Banchs et al., 2012). However, the use of virtualization techniques in wireless mesh networks (WMNs) has thus far not been adequately studied even though, as we will show, it can introduce new business scenarios for network operators.

WMNs are known for their ability to extend the reach of existing networks (Azcorra et al., 2009). In traditional concepts such a mesh cloud is governed by a single mesh network operator (MNO). We propose a business scenario in which network virtualization is used to divide the mesh resources among multiple virtual network operators (VNOs) (Figure 1). Each VNO has dedicated resources in the form of a Virtual Network (VN). The VN extends from traffic entry points (either WiFi APs or, in general, Access Routers, ARs) to gateways (GWs) leading to the Internet core network. Each VN has statistically guaranteed QoS parameters. The advantage of this outsourcing approach is that each VNO can focus on providing a service to its end-users, leaving the network administration to the MNO. This leads to the lowering of the operating expenditure of VNOs. Furthermore, each VN can be tailored to both operator and service requirements.

In this paper we describe VIMENO: a Virtual wireless Mesh Network architecture for Operators. This architecture provides the aforementioned virtualization features. Our approach is based on WiFi devices (IEEE 802.11, 2012), which are known for their large popularity, low cost, license-free operation, high extendibility, and constant improvement through new IEEE 802.11 amendments. Adopting an approach based on statistical packet multiplexing is in contrast with existing solutions, which are based on circuit switching (most commonly TDMA). However, our approach ensures greater flexibility which is important when dealing with both bursty traffic as well as varying radio channel conditions. Furthermore, our approach is the first virtualization-enabled WMN architecture designed for operators: previous efforts focused either on networks for research (Shrestha et al., 2008) or on providing community network access (Matos et al., 2011) or were proposed for ad hoc networks (Dedecker et al., 2011).

The architecture described in this paper is an ini- 
tial study of the concept of virtual WMNs for operators. We would like to share this concept with the reserach community to stimulate discussion on the numerous research challenges which we have identified. We plan to implement VIMENO to further study these issues and provide suitable solutions.

The remainder of this paper is organized as follows. In Section 2 we discuss the business aspects of our approach. In Section 3, we provide an overview of the node types forming the network architecture as well as the relevant networking modules. In Sections 4 to 7 we discuss the most important features of VIMENO: network monitoring, resource separation, traffic shaping, and routing, respectively. The conclusions and future work are summarized in Section 8.

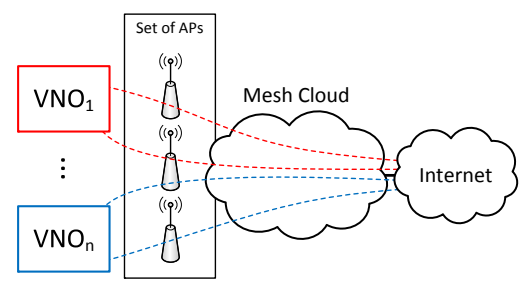

Figure 1: Concept of the proposal.

\section{CONTRACT}

The network architecture and its functionality is determined by the contract agreed upon between the MNO and its customers, the VNOs. The main task of the MNO is to ensure the contract execution for all VNOs and in the case of temporary resource scarcity (due to, e.g., congestions, degradation of propagation conditions, or failures) perform appropriate reconfigurations to provide fair, or in accordance with an agreed policy, resource distribution. Moreover, we define a method for flexible and dynamic resource distribution according to generated traffic as a part of the VIMENO architecture's self-organising functionality.

Multiple detailed schemes for the contract can be considered, however, for the purpose of our investigations we have decided to define a flexible one to provide smart resource distribution within VNs and create a solid base for further developments. Thus, the VNO buys uplink and downlink throughput $\left(R^{U L}, R^{D L}\right)$ and specifies a set of Mesh Access Nodes $\left\{M A N_{n}\right\}$, where $n \in\left(0, N_{M A N}\right]$ and $N_{M A N}$ is the total number of MANs in the network, on which this VN will be available. The throughput guarantee is statistical with a given rate probability function, which is in line with current mobile network operator business models for services based on packet data transmission. It is up to the network planning process and in particular its dimensioning phase to ensure that there are, statistically, enough resources to meet the contract demands. We can determine the relation between contract commitments and the network capacity as follows:

$$
\begin{aligned}
& \sum_{i=1}^{N_{V N O}} R_{i}^{U L} \leq C^{U L}(1+\varepsilon), \\
& \sum_{i=1}^{N_{V N O}} R_{i}^{D L} \leq C^{D L}(1+\varepsilon),
\end{aligned}
$$

where $R_{i}^{U L}$ and $R_{i}^{D L}$ is the contracted throughput in the uplink and downlink direction, respectively, for the $i$ th operator; $N_{V N O}$ is the number of VNOs, $C^{U L}$ and $C^{D L}$ are the uplink and downlink network capacity, respectively; and $\varepsilon$ is the capacity under-provisioning factor. These equations reflect our assumption that typically the total load of the WMN will be smaller than its capacity, and, therefore, we can assume statistical resource sharing.

Based on the signed contracts, the MNO is responsible for setting up VNs and providing connectivity within the mesh network. The access part of the network is separated between VNOs by having each AP located at a MAN broadcast individual SSIDs for each VN. VNs are also treated independently by the remaining physical mesh infrastructure. Thus, multiple routing protocol instances must be involved to define transmission paths. The physical network architecture is a mesh, however all paths are established between MANs and Mesh Gateways (MGW) to provide Internet access, which results in the functional architecture of multiple trees. Periodically, or in response to radio link quality degradation or failures in the network, the paths can be modified by the routing protocol. Hence, some paths can also be redirected to other MGWs. Such an operation may result in load variation at the MGW and other intermediate mesh nodes. Moreover, the load can be distributed unequally among VNs, since VNOs define independent sets of MANs and independent throughput contracts. "If there is a risk of exceeding the maximum capacity of any link of an MGW (or any other mesh node), the throughput values currently configured for particular VNOs must be fairly decreased at that node. This means that each mesh node must know the proportion of particular VN load requirements that are to be met. This can be reported by the MANs or MGWs currently attached to the mesh node or by a central dedicated management entity that is to serve as a central resource distribution coordination point. These issues are further discussed in Sections 5 and 6.

In order to simplify the description, we assume that all the connections in the mesh network have been 
configured. We therefore bypass the initial bootstrapping stage. Additionally, we exclude mobility issues (i.e., the movement of VNO clients between MANs) from our field of study. Finally, we assume that all traffic flows initiate or terminate, from the mesh network perspective, in the MANs and MGWs.

\section{NETWORK AND NODE ARCHITECTURE}

Figure 2 presents a detailed view of the network architecture in VIMENO, highlighting the data paths in a given example topology. In the following, we give a brief overview of the elements presented in this figure. There are three mesh node types in the network: (i) Mesh Gateway (MGW) - interconnects the mesh and the core networks using either wireless or fixed links, (ii) Mesh Access Node (MAN) - offers network access to end users, typically equipped with at least two wireless interfaces: one for providing network access and the other for connecting to the mesh network, (iii) Mesh Relay Node (MRN) — interconnects MANs with MGWs and relays user traffic, typically equipped with at least two wireless interfaces: one in the uplink and one in the downlink data transfer direction, respectively. In practice, to achieve better mesh network performance, it is recommended that each MRN have at least three wireless interfaces.

Each mesh node (Figure 3) contains the following building blocks: (i) wireless interfaces - typical IEEE 802.11 interfaces, (ii) virtual wireless interfaces - to support the various VNs transported in the mesh network, (ii) schedulers - to properly plan the transmission from each virtual interface, (iv) traffic shapers - to limit the amount of traffic transported in the network, they are present only in the ingress nodes: MANs and MGWs for the uplink and downlink directions, respectively, (v) monitoring module - to measure and estimate current PHY and MAC parameters, (vi) routing module - to orchestrate traffic forwarding within the network, a separate instance is executed for each VN.

In principle, the network operates as follows. Traffic from VNs is separated using virtual interfaces and appropriately configured schedulers (Section 5). The traffic shapers are configured in such a way as to fulfil the contract and minimise the traffic transported in the mesh network (Section 6). Each VN employs a separate routing protocol (Section 7). All these mechanisms are supported by passive network monitoring (Section 4). In the following sections, we describe each mechanism in detail.

\section{MONITORING}

Since the parameters of wireless links cannot be determined in advance, they need to be estimated after the setup of wireless links. Additionally, during network operation, the parameters can change due to fluctuations of the radio propagation conditions or interference from other networks. Typical operating system tools do not provide reliable estimates on the available and used data rates on the wireless links. The only possibility to obtain such information is to use a dedicated monitoring mechanism. In the VIMENO architecture we define the Monitoring Module (MM), which provides a passive monitoring service able to measure several parameters related to radio channel conditions, capabilities of neighbouring nodes and IEEE 802.11 MAC parameters estimation. MM provides precise network measurements without disrupting network operation.

The results of MM measurements are utilized mainly by the routing module, shapers, and schedulers. During normal network operation the most important parameters that have to be delivered by the MM for each link are: the available data rate, the data rate used by each VN, delay, jitter, and packet loss ratio. The MM module performs measurements at the PHY and MAC layers within the time-scale of microseconds, based on all types of 802.11 frames (data, management, and control). To ensure a comprehensive view of the current wireless channel conditions, including traffic from networks of other operators, the promiscuous mode of operation of wireless network cards is used. The use of passive mode means that all measurements are performed along with the normal activity of the wireless card.

The MM works on a frame level, i.e., all frames sent and received by each network interface must be examined by the MM functions. This imposes high requirements on the implementation effectiveness of the frame analysis (i.e., the limited computational power available at the nodes should be taken into account). In the Linux operating system the abovementioned requirements force an implementation of $\mathrm{MM}$ as a kernel module closely interworking with the mac80211 wireless framework (mac80211, 2013).

\section{RESOURCE SEPARATION}

In VIMENO, network virtualization is realized differently in the access and mesh parts of the network. This is illustrated in Figure 4. The differences between the two virtualization methods (link and interface) are subtle. We discuss them below. 


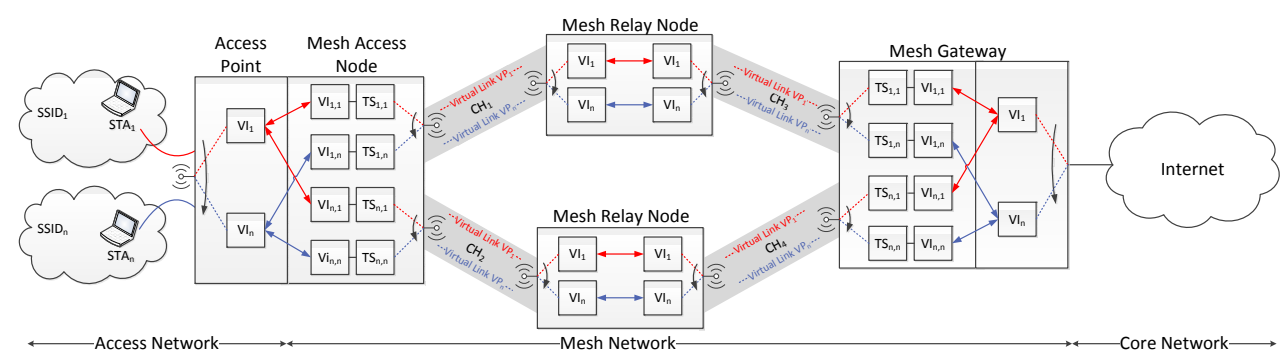

Figure 2: Network architecture with basic elements.

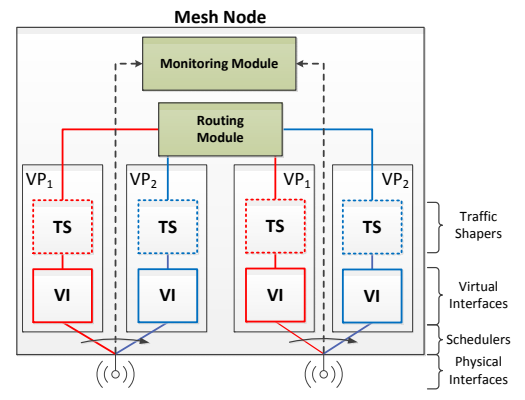

Figure 3: Architecture of the mesh node.

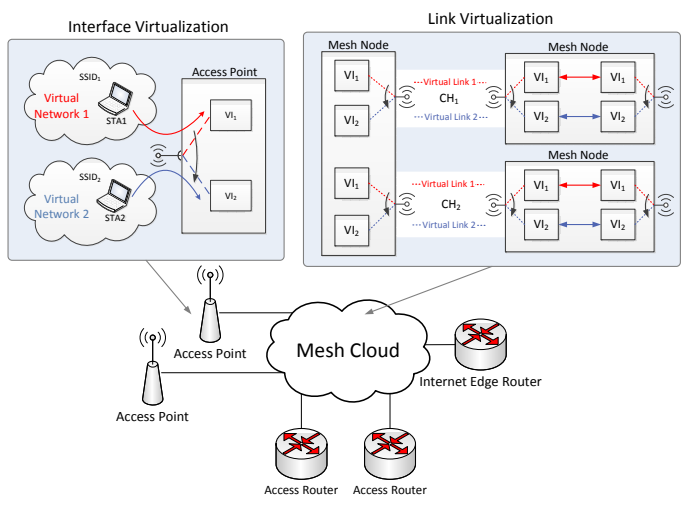

Figure 4: Separation between virtual networks.

In the access part of VIMENO, the VNs are separated using standard interface virtualization. Therefore, the wireless interface of each AP (at the MAN) is virtualized and a separate virtual interface (VI) is provided for each VNO. This allows separation between VNOs by setting a different SSID for each of them. This type of interface virtualization has been described in numerous papers and, therefore, we refer the reader to (Chandra and Bahl, 2004; Smith et al., 2007; Rivera and Zucci, 2010; Al-Hazmi and de Meer, 2011; Braham and Pujolle, 2011) for further details. Currently, we assume standard 802.11 fairness in scheduling between VIs. However, in the future we foresee remote configuration of the client node MAC parameters by the AP.

In the mesh part of VIMENO, the VNs are separated using link virtualization. Therefore, a sepa- rate virtual link is provided for each VNO on a single wireless channel ${ }^{1}$. The separation of traffic (i.e., packet flows) between VNs is based on statistical packet scheduling (described below). Such a solution is cheap, scalable, flexible, does not require synchronization, and finally, it is in line with the packet nature of the Internet.

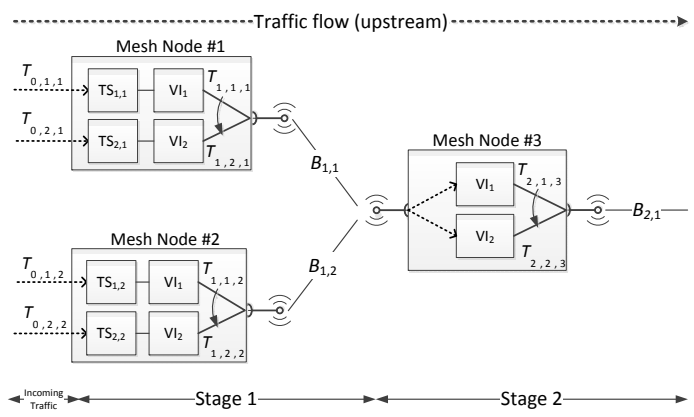

Figure 5: Generalized intra-mesh scheduling assuming two VNOs.

The generalized intra-mesh scheduling method is illustrated in Figure 5. In principle, the goal of the scheduler's operation is, in the absence of sufficient resources, to fairly divide the available link capacity among the VNs. This is performed in proportion to current traffic shaper settings. At the first stage $(i=1)$, i.e., at the MAN or MGW, the scheduler is configured so that the outgoing traffic rate (in b/s) of each VI $j$ of the $k$-th mesh node is set as follows

$$
T_{1, j, k}=\min \left(\frac{T S_{j, k}}{\sum_{h=1}^{N_{V N O}} T S_{h, k}} B_{1, j}, T_{0, j, k}\right),
$$

where $T S_{j, k}$ is the setting of the $j$-th traffic shaper of the $k$-th mesh node, $N_{V N O}$ is the number of VNOs, and $B_{1, j}$ is the capacity of the outgoing link of the $k$ th mesh node. This means, that under non-saturation $T_{1, j, k}=T_{0, j, k}$ (i.e., the current incoming traffic rates correspond to the outgoing traffic rates). Additionally, under saturation $T_{1, j, k}=\frac{T S_{j, k}}{\sum_{h=1}^{N V N O} T S_{h, k}} B_{1, j}$, which means

\footnotetext{
${ }^{1} \mathrm{~A}$ channel allocation scheme is out of the scope of this paper. We refer the reader to (Lv et al., 2012) for an exemplary OFDM-based scheme.
} 
that the outgoing traffic rate is scaled according to the capacity of the outgoing link of the $k$-th mesh node and the current settings of appropriate traffic shapers.

At stages $i>1$ the outgoing traffic rate from each VI $j$ of the $k$-th mesh node is dependent on the rate configurations of the traffic shapers located at the upstream ingress nodes:

$$
\begin{aligned}
& T_{i+1, j, k}=
\end{aligned}
$$

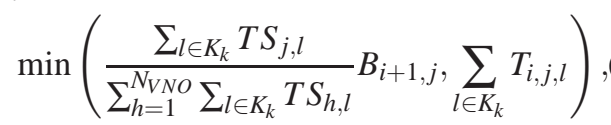

where $K_{k}$ is the set of upstream one-hop neighbors of $k$. Under non-saturation all traffic arriving to the $k$-th mesh node is transmitted to the next stage $\left(T_{i+1, j, k}=\sum_{l \in K_{k}} T_{i, j, l}\right)$. Under saturation, the outgoing traffic rate from the $k$-th mesh node is scaled according to the value of the capacity of the outgoing link $\left(T_{i+1, j, k}=\frac{\sum_{l \in K_{k}} T S_{i, j, l}}{\sum_{h=1}^{N_{V N O}} \sum_{l \in K_{k}} T S_{i, h, l}} B_{i+1, j}\right)$. Such an approach requires the dissemination of current TS rate configurations, but assures fairness in relation to both the contracts as well as the current VNO traffic requirements.

In order to discriminate between VNs VLAN tags are employed (IEEE 802.1Q, 2011). They are placed after the IEEE 802.11 header in each frame. Such a solution is flexible, backward compatible, and allows straightforward implementation in wireless devices.

\section{TRAFFIC SHAPING}

In the VIMENO architecture it is necessary to assure that the throughput contracts are not breached, i.e., that the traffic admitted into the mesh network for each VN does not exceed the VNOs contracted throughput values. This is achieved through the appropriate configuration of traffic shapers, which are placed at the ingress nodes. At the MAN (MGW), they guarantee that the uplink(downlink) contracted throughput is within limits.

To ensure that contracts are fulfilled and to provide flexible resource distribution, dedicated signalling is required to periodically inform traffic shapers about current data rates for VNs. This can be performed using a centralized or distributed approach. The final solution for the signalling architecture should be selected according to the required functionality, efficiency, and scalability of the network. For the purpose of VIMENO deployment we assume a centralized management approach. A dedicated server is located on the outside of the considered mesh network (physically, it may be at one of the MGWs or in the core network). As future work, we will consider a distributed management approach which is required for large mesh networks.

We assume that the contracted throughput values will be fairly divided among the MANs and MGWs for each VNO. This requires an appropriate configuration of shapers located at these nodes. This configuration is performed using the following algorithm. First, the central server sends updates to the traffic shapers located at the MANs and MGWs with their initial configuration. These updates contain the throughput values for the uplink and downlink directions, as contracted by each VNO, divided by the selected number of MANs (for uplink) and MGWs (for downlink). Next, the obtained throughput values are set as the shaping rates for each VN at both MANs and MGWs. This means that for a given VN, the shaping rates are equal for each selected MAN as well as for each selected MGW. In order to effectively use the available resources in the mesh network, for each $\mathrm{VN}$ the shapers are independently controlled using the AIMD (Additive Increase, Multiplicative Decrease) algorithm (Crisostomo et al., 2005). This algorithm uses information about the resources available at the MANs and MGWs as input to provide a configuration which assures proportional sharing of resources between VNs as feedback.

After the initial configuration of shapers, if there is both on-going traffic as well as available resources, the shaping rates can be reconfigured in the following way. Every time interval $t$, the rate of each shaper is independently increased for each $\mathrm{VN}$ by an increment of $c \mathrm{~kb} / \mathrm{s}$ until a given threshold for each MAN or MGW is exceeded (this threshold is set to $95 \%$ of the available resources to ensure that the network operates in non-saturation). The sum of the configured shaping rates at MANs and MGWs for each VN cannot be larger than the contracted throughput values (either for the uplink or downlink direction). It should also be noted, that when the shaping rate substantially exceeds the actual rate, there is the risk of transmitting data bursts without due control, which may affect link utilization. In order to avoid this problem, the rate controller located at the same entity monitors the current transmission, and regulates the shaping rate in order to not exceed the actual rate in more than a gap percent of the actual rate. The AIMD shaping algorithm is given in Algorithm 1 in the form of pseudo code. 


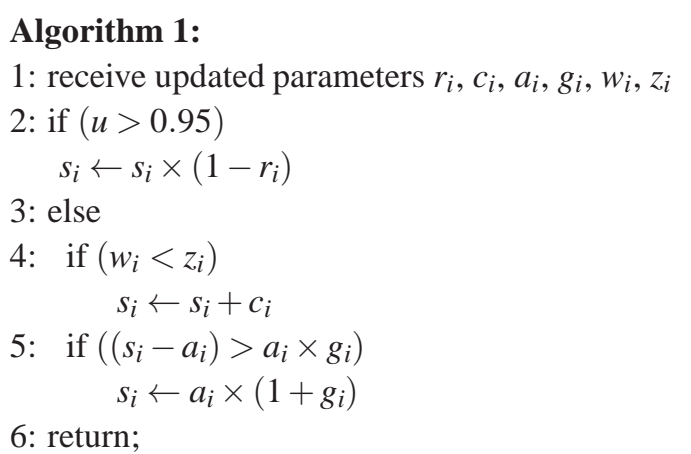

where: $u$ is the link utilization; $s_{i}$ is the shaping rate of shaper $i$ for the $i$-th $\mathrm{VN} ; w_{i}$ is the sum of actually configured upstream shaping rates for the $i$-th $\mathrm{VN} ; z_{i}$ is the sum of the contracted upstream throughput values for the $i$-th $\mathrm{VN} ; r_{i}$ is the multiplicative decrease factor for the $i$-th $\mathrm{VN} ; c_{i}$ is the additive increase increment for the $i$-th $\mathrm{VN} ; a_{i}$ is the actual outgoing traffic rate of the shaper for the $i$-th $\mathrm{VN} ; g_{i}$ is the maximum gap of $r_{i}$ concerning $a_{i}$ for the $i$-th VN.

Moreover, if there is no traffic in the $\mathrm{VN}$ at specific MAN or MGW, the shaping rate at MAN and/or MGW for this VN can be decreased down to the minimum defined throughput value $m_{i}$ using the following rule: every time interval $t$, the rate of each shaper is decreased by a multiplicative factor $r_{i}$.

It could also happen that a new traffic flow appears in a $\mathrm{VN}$ while the shaping rate for this $\mathrm{VN}$ was decreased at specific MAN and/or MGW to the level of $m_{i}$. If this is the case, a reconfiguration procedure should be executed. First, the server checks the actual value of $w_{i}$. If $w_{i}<z_{i}$ and there are enough resources to increase the shaping rate at specific MAN and/or MGW, it is increased using Algorithm 1. In any other case, to prevent saturation conditions and assure proportional and fair distribution of resources for each VN, the server sends to all MANs and MGWs a request to proportionally decrease the shaping rate for all VNs by a multiplicative factor $r_{i}$. This procedure is repeated until there are enough resources to start the process of the shaper reconfiguration. Second, the shaping rate at newly used MAN and/or MGW for this VN is increased proportionally to the values of throughput configured at all other MANs and/or MGWs of the same VN (proportionally to the contracted throughput values of each VN) using AIMD.

To summarize, the server plays an important role in the process of guaranteeing VN throughput. It collects information about the contracted upstream throughput values, existence of VN traffic at each MAN and MGW, available resources at MANs and MGWs, and the actual rate of shapers for each VN. This allows for efficient and fair distribution of the available mesh network resources between the VNs.

\section{ROUTING}

Routing plays a leading role in the data exchange within WMNs, especially in terms of service availability and reliability. For a large number of MRNs in a WMN topology, the role of the routing protocol is even more important, because it has to deal with the complexity of a multi-path topology. Additionaly, taking into account the dynamics caused by the time varying wireless links, it can be concluded that only a dynamic, link-state routing protocol, supported by a constant network monitoring, is capable of providing a reliable service for VNOs.

The most important objectives of a dynamic routing protocol in WMNs are: (i) remote network discovery and information sharing among network nodes, (ii) determination of the best path towards the destination (between MANs and MGWs), (iii) maintenance of routing information and routing table updated upon topology or link state change, and (iv) ability of traffic rerouting along a new path in case of transmission condition changes.

The most essential component of each routing protocol is the routing protocol metric, since it drives the best path selection procedures. Currently, a variety of different metrics for WMNs exist. They are mainly adapted from mobile ad-hoc networking. The most typical metrics proposed for WMNs are the following (Bin Ngadi et al., 2012): ETX (EXpected Transmission Count) with its modifications, ETT(Expected Transmission Time), WCETT (Weighted Cumulative ETT), RARE (Resource Aware Routing for MESH), EDR (Expected Data Rate), CATT (Contention Aware Transmission Time), and ACRM (Airtime Cost Routing Metric, the default metric for the IEEE 802.11s standard).

Unfortunately, none of these typical metrics could be adopted without change to provide a complete radio link state description, taking into account the virtualization concept, as this requires a more detailed image of a variety of cross-layer parameters (L1/L2/L3). Such a cross-layer approach allows to cover not only the radio channel state (number of transmitting nodes or estimated free bandwidth), but extends it with a specific link parameters (delay, average throughput, etc.).

Since a simple mapping of the existing wireless routing protocol metrics into virtualized WMNs is not sufficient, a question arises: what kind of metric could satisfy the requirements of WMN reliability with respect to the demands of VNOs? It seems to be evident that only a cross-layer approach together with a composite metric design addresses such a problem.

The commonly known example of a composite 
metric is the one used by the EIGRP routing protocol (EIGRP, 2005). It is formulated as follows:

$$
M=\frac{\left(K_{1} \times B+\frac{K_{2} \times B}{256-L}+K_{3} \times D\right) K_{5}}{R+K_{4}},
$$

where $M-$ metric, $B-$ bandwidth (in bits per second), $L-$ load, $D-$ delay, $R$ - reliability. Additionally, $K_{1}$ to $K_{5}$ are the cumulative weights. By default $K_{1}$ and $K_{3}$ are set to 1 , while the others are set to 0 . The EIGRP protocol uses the minimum bandwidth on the path towards the destination network and the total delay to calculate its routing metric. The load component reflects link saturation, while the reliability component represents the measure of the likelihood that a link will fail. A detailed description of the EIGRP metric can be found in (EIGRP, 2005).

In case of time varying wireless links, the formula of a composite metric is not so evident as for EIGRP, since we cannot rely on the stability of the bandwidth and delay values. ${ }^{2}$ Therefore, the proposed VIMENO composite metric should be based on components selected from the following four generic groups: (i) Basic: hop count, estimated maximum available bandwidth; (ii) Quality of Service: link capacity, average transmission rate, aggregated perhop and overall delay, jitter, BER; (iii) Interference: number of nodes operating on the same channel, SNR ratio, RxPwr ratio; (iv) Route Stability: considered as the radio link stability with respect to a rate utilization histogram analysis and link availability in a long timescale. The following parameters have been selected as the most representative elements of the VIMENO composite metric: (i) Bandwidth estimations: ratio of the required, contractual, bandwidth or average available throughput (per VNO) compared to the estimated free channel bandwidth; (ii) Interference: number of nodes transmitting on a single radio channel; (iii) Route stability: multi-rate utilization and link availability.

These parameters will be provided by the Monitoring Module operating on all the physical interfaces of the mesh node. However, the exact definition of the final VIMENO composite metric formula requires a detailed mathematical and simulation analysis, which is beyond the scope of this paper.

Importantly, the VIMENO architecture is able to support traffic separation between VNOs. This requires that at each physical radio link a dedicated virtual link is assigned for a single VNO. The virtual link is then established between a set of virtual interfaces on the physical ones at neighbouring WMN nodes.

\footnotetext{
${ }^{2}$ Originally, both parameters, bandwidth and delay, in the EIGRP metric are assumed to be static.
}

The set of virtual links composes a VN topology for a VNO. To assure end-to-end connectivity, a dedicated link-state routing protocol is required to operate separately for each VN topology.

The main goal of the routing protocol is to provide optimal path selection between MANs and MGWs and activate a rerouting procedure in case of degradation of radio conditions. The rerouting process assumes that multiple paths exist between MANs and MGWs. To satisfy this assumption, during the VNO network setup phase, the virtual interfaces dedicated to a specific VNO should be configured. Additionally, virtual links should be set up over all physical ones, so as not to limit the possibilities of alternative path selection.

Due to the limitation of the radio resources, we cannot assume resource over-provisioning at a similar level as it is possible in fixed networks, but some over-provisioning may need to be considered to avoid link congestion. In the case of virtualized WMNs link congestion may be caused by synchronization of rerouting decisions within the same physical node for different VNOs. To avoid this situation, the value of the composite metric should be calculated separately for each VN and the metric computation algorithm should avoid synchronization of the rerouting process. As a result, a slight time shift in the signalling of radio link parameter degradation to each VNO routing protocol is considered. This helps to avoid the problem of path flipping between interfaces at the same physical node for all existing VNOs at the same time. The selection of the VN which should be triggered to activate the rerouting procedure may be based on the VNO contract with respect to the required QoS parameters (delay, jitter, etc.), business-level parameters (VNO priority), currently used bandwidth, etc.

At the first stage of the VIMENO architecture development, for simplicity and taking into account that multiple VNO networks exist in a single WMN node, load balancing takes place at the VNO level. Therefore, we do not consider multi-path routing inside VNO networks. The analysis of multi-path routing is planned as our future work.

\section{CONCLUSIONS AND FUTURE WORK}

This article has presented an initial study of VIMENO, the first virtual wireless mesh network architecture for operators. The novelties proposed in the article include: definition of a new business scenario in which network virtualization is used to divide the mesh resources among multiple VNOs; distinc- 
tion between interface and link virtualization for the access and mesh network parts, respectively; architecture of a virtualized mesh node; virtualization of the mesh network composed of intra-mesh scheduling combined with the assignment of VLAN tags; shaping technique based on the throughput metric, providing upstream and downstream guarantees for VNOs; and link-sate routing protocol, composed of different instances for each VNO. A promising advantage of the proposed architecture (in contrary to the previous TDMA-based solutions) is the fact that it is in line with the packet nature of the Internet.

Our future work will be directed towards the implementation of VIMENO in real wireless devices. This will allow testing and optimizing the proposed solutions. Future enhancement of VIMENO is also planned in order to make the architecture even more suited to various possible requirements of wireless network operators and services. The following networking challenges will be considered: design of alternative routing solutions supporting the virtualization concept, definition of the formula for the routing composite metric, distributed network management and traffic shaping, wireless network bootstrapping, appropriate load balancing, network reconfiguration and rerouting, network resilience and survivability, security of signalling data, and quality of service (QoS) provisioning. They will be designed taking the perspective of VNOs into account. Obviously, an introduction of new functionalities will require rethinking of the currently proposed solutions and protocols. E.g., the introduction of QoS provisioning will impact the settings of traffic shapers and schedulers.

\section{ACKNOWLEDGEMENTS}

This work has been carried out as a part of a project financed by the Polish National Science Centre (decision no. DEC-2011/01/D/ST7/05166)).

\section{REFERENCES}

Al-Hazmi, Y. and de Meer, H. (2011). Virtualization of 802.11 interfaces for wireless mesh networks. In Proc. of WONS 2011.

Azcorra, A., Banniza, T., Chieng, D., Fitzpatrick, J., VonHugo, D., Natkaniec, M., Robitzsch, S., and Zdarsky, F. (2009). Supporting carrier grade services over wireless mesh networks: The approach of the European FP-7 STREP CARMEN. Commun. Mag., 47(4):1416.

Banchs, A., Serrano, P., Patras, P., and Natkaniec, M. (2012). Providing Throughput and Fairness Guaran- tees in Virtualized WLANs Through Control Theory. Mob. Netw. Appl., 17:435-446.

Bin Ngadi, M. A., Ali, S., Abdullah, A. H., and Khokhar, R. H. (2012). A taxonomy of cross layer routing metrics for wireless mesh networks. Journal on Wireless Communications and Networking, 2012(177).

Braham, O. and Pujolle, G. (2011). Virtual wireless network urbanization. In Network of the Future (NOF), 2011 International Conference on the, pages 31-34. IEEE.

Chandra, R. and Bahl, P. (2004). MultiNet: Connecting to multiple IEEE 802.11 networks using a single wireless card. In Proc. of INFOCOM 2004.

Chowdhury, N. and Boutaba, R. (2010). A survey of network virtualization. Comp. Net., 54:862-876.

Crisostomo, S., Sargento, S., Natkaniec, M., and Vicari, N. (2005). A QoS architecture integrating mobile adhoc and infrastructure networks. In Proc. of AICCSA 2005.

Dedecker, P., Hoebeke, J., Moerman, I., Moreau, J., and Demeester, P. (2011). Network virtualization as an integrated solution for emergency communication. Telecommunication Systems, pages 1-18.

EIGRP (2005). Enhanced Interior Gateway Routing Protocol. http://www.cisco.com/en/US/tech/tk365/ technologies_white_paper09186a0080094cb7. shtml.

IEEE 802.11 (2012). IEEE Standard for Information technology-Telecommunications and information exchange between systems-Local and metropolitan area networks-Specific requirements-Part 11: Wireless LAN Medium Access Control (MAC) and Physical Layer (PHY) Specifications, March 2012.

IEEE 802.1Q (2011). IEEE 802.1Q-2011 — IEEE Standard for Local and metropolitan area networks-Media Access Control (MAC) Bridges and Virtual Bridged Local Area Networks.

Lv, P., Wang, X., and Xu, M. (2012). Virtual access network embedding in wireless mesh networks. Ad Hoc Networks, 10(7):1362-1378.

mac80211 (2013). Linux wireless (IEEE-802.11) subsystem. http://linuxwireless.org.

Matos, R., Sargento, S., Hummel, K. A., Hess, A., Tutschku, K., and de Meer, H. (2011). Context-based wireless mesh networks: a case for network virtualization. Telecommunication Systems, 51:1-14.

Rivera, A. D. and Zucci, W. (2010). Virtualization of wireless network interfaces Wi-Fi IEEE 802.11. In Proc. of TELE-INFO 2010.

Shrestha, S., Lee, J., and Chong, S. (2008). Virtualization and slicing of wireless mesh network. In Proc. of CFI 2008.

Smith, G., Chaturvedi, A., Mishra, A., and Banerjee, S. (2007). Wireless virtualization on commodity 802.11 hardware. In Proc. of ACM WiNTECH 2007. 